\title{
Modeling and Inferring in Science
}

\author{
Emiliano Ippoliti ${ }^{*}$, Thomas Nickles ${ }^{\dagger}$ and Fabio Sterpetti ${ }^{\ddagger}$ \\ ${ }^{*}$ Department of Philosophy, Sapienza University of Rome, Italy \\ emi.ippolitiegmail.com \\ † Department of Philosophy, University of Nevada, Reno, USA \\ nickles@unr.edu.it \\ * Department of Philosophy, Sapienza University of Rome, Italy \\ fabio.sterpetti@uniromal.it
}

\begin{abstract}
Science continually contributes new models and rethinks old ones. The way inferences are made is constantly being re-evaluated. The practice and achievements of science are both shaped by this process, so it is important to understand how models and inferences are made. But, despite the relevance of models and inference in scientific practice, these concepts still remain controversial in many respects. The attempt to understand the ways models and inferences are made basically opens two roads. The first one is to produce an analysis of the role that models and inferences play in science. The second one is to produce an analysis of the way models and inferences are constructed, especially in the light of what science tells us about our cognitive abilities. The papers collected in this volume go both ways.
\end{abstract}

Science continually contributes new models and rethinks old ones. The way inferences are made is constantly being re-evaluated. The practice and achievements of science are both shaped by this process, so it is important to understand how models and inferences are made.

Despite the relevance of models and inference in scientific practice, these concepts are not only multifaceted but also in some sense their definition, role and purpose still remain controversial in many respects.

Let us start with the notion of model. Frigg and Hartmann, for instance, state that:

Models can perform two fundamentally different representational functions. On the one hand, a model can be a representation of a selected part of the world (the 'target system'). [...]. On the other hand, a model can represent a theory in the sense that it interprets the laws and axioms of that theory. These two notions are not mutually exclusive as scientific models can be representations in both senses at the same time. ${ }^{1}$

1 Frigg and Hartmann 2012, § 1. 


\section{PENULTIMATE DRAFT - PLEASE CITE THE PUBLISHED VERSION}

To appear in: Models and Inferences in Science, Ippoliti, E., Sterpetti, F. and Nickles, T. (eds.), Springer.

It seems that the concept of 'model' is so wide that it cannot be grasped by means of a single, clear definition, and thus its meaning is still controversial. In effect, there are several definitions of what models are, which often sharply diverge (see e.g. Krause and Bueno 2007, p. 187). For example, Bailer-Jones states that a "model is an interpretative description of a phenomenon that facilitates access to that phenomenon," and that interpretative descriptions may rely "on idealizations or simplifications or on analogies to interpretative descriptions of other phenomena." Moreover, models can "range from being objects, such as a toy airplane, to being theoretical, abstract entities, such as the Standard Model of the structure of matter and its fundamental particles" (Bailer-Jones 2009, p. 1-2). Along this line, models may be easily conceived as instruments, 'neither true nor false': instead, they are useful heuristic devices, which often are effective even when they are 'false'.

On the contrary, it has been argued that models cannot be interpreted as useful heuristic devices, because if "theories are vehicles of scientific knowledge, then so too must models be" (Suppe 2000, p. S109). The reason for such a claim is that if knowledge is intended as being related to the truth, and theories are vehicles of knowledge, models of such theories have to be true, nor just metaphor-like or heuristic devices. In fact, in model theory models of a theory make true the axioms of such theory. Since those who adopt the semantic view of theories adopt the model theoretic concept of 'model', they cannot think of models as heuristic devices (Morrison 2009). For example, Suppes claims that "the concept of model in the sense of Tarski may be used without distortion and as a fundamental concept" in scientific and mathematical disciplines, and that "the meaning of the concept of model is the same in mathematics and the empirical sciences" (Suppes 1961, p. 165). According to Suppe, "Suppes' claim is that the Tarski concept of a model is a common formal framework for analysis of various uses of models in science and mathematics" (Suppe 2000, p. S111).

Many authors have criticized this conflation of different senses attached to the term 'model' (Thomson-Jones 2006). But the problem is that if we decouple the concept of model used in model theory from that used for heuristic purposes in scientific practice, then it is difficult to maintain some of the traditional realist claims about the truth of our best scientific theories that many philosophers of science subscribe to. Indeed, the best tools to describe the idea that our best theories correctly 'correspond' to the world have been for a long time Tarski's theory and the notion of 'isomorphism' (da Costa and French 2003).

In effect, the move of denying the identity of the concept of model used in mathematics and that used in scientific practice, by trying to develop a more subject- and context-dependent notion of model centered on the notion of 'representation' instead of that on that of 'isomorphism', has faced two main objections.

First, there is the argument from realist-minded philosophers that such a motive implies or at least invites a sort of instrumentalism that is not able to preserve the objectivity of science, and thus risks to open the door to skepticism or relativism. Second, again coming from some realist philosophers, is that the notion of representation used by the instrumentalists may be in its turn accounted for in terms of isomorphism, and so that the notions of model used in mathematics and in scientific practice 


\section{PENULTIMATE DRAFT - PLEASE CITE THE PUBLISHED VERSION}

To appear in: Models and Inferences in Science, Ippoliti, E., Sterpetti, F. and Nickles, T. (eds.), Springer.

are not really distinct and may be in the ultimate analysis reduced to one (French 2003).

As concerns the notion of inference, its role, nature and purpose are at stake as well, since the orthodox viewpoint put forward by the analytic tradition, modeled on mathematical logic, displayed more and more weaknesses, especially in the attempt to account for the growth of knowledge (see e.g. Cellucci 2013; Ippoliti 2014). For an increasing number of philosophers, this problem requires a completely new approach to the concepts of knowledge and inference, both internally and externally.

More specifically, the standard view of the notion of inference is "formulated by Hintikka and Sandu as follows: 'Inferences can be either deductive, that is, necessarily truth preserving, or ampliative, that is, not necessarily truth preserving"' (Cellucci 2013 , p. 295). Such a distinction is internally inadequate, since it does not produce a cogent classification of the various kinds of inference. In particular it does not work for abduction. In fact, abduction, as many people understand it, is neither ampliative nor truth preserving, and hence it is a counter-example to this standard way of conceiving inferences. If we accept the orthodox distinction between deductive rules (truth preserving) and ampliative rules (non-deductive, and hence not truth preserving), it turns out that abduction "belongs to a different category because, on the one hand, like deductive rules, it is non-ampliative, but, on the other hand, unlike them, it is not truth preserving" (Ibidem, p. 302).

On the other side, the standard view is unsatisfactory also externally, that is, with respect to the nature, role and purposes of knowledge. For, in the end, it does not account for the growth and ampliation of knowledge. Mathematical logic, the model of the analytic tradition, is a tool explicitly designed to systematize and justify what is already known. It does not aim at producing genuinely new knowledge, since its purpose is to provide a secure foundation for our scientific knowledge, in particular mathematics; and the method to do that is the deductive method.

First, mathematical logic fails as a means of justification, in virtue of a careful reading of the limitative results in general, and of the consequences of Gödel's incompleteness theorems in particular (see Cellucci 2013).

Second, the analytic tradition and mathematical logic essentially draw on a restriction on the scope of logic, and hence inference, with respect to Plato, Aristotle, Descartes and Kant, which turned out to be detrimental to its role in the scientific research.

A promising way out to these difficulties is to approach the notion of inference using different notions, namely the one of containment instead of truth preservation and the one of vindication instead of validation. We will sketch here the former.

We can produce a more cogent classification of inferential rules in terms of ampliative and non-ampliative ones. The former, like induction or analogy, are such that their conclusions are not contained in the premises, the information in the conclusion goes beyond the information in the premises. And because of that they can go wrong, even if they have heuristic power. The latter, like the deductive rules, are such that the conclusion is contained in the premises, that is, the conclusion either is literally a part of the premises, or entails nothing that is not already entailed by the premises. For instance, in Modus Ponens the conclusion B is literally included in the premis- 
es $\mathrm{A}$, and $\mathrm{A} \rightarrow \mathrm{B}$. Therefore, deductive rules, as non-ampliative rules, have no heuristic power. For a new idea (B in this case) must already be available before the inference can be constructed. It is not, therefore, an inference to $\mathrm{B}$ as new knowledge. It goes without saying that it does not mean that they are useless. As a matter of fact, since the conclusion of a deductive rule makes explicit all or part of what is contained in the premises, it enables us to establishing that its conclusion is plausible, facilitating the comparison of the premises with experience.

The bottom line here is that there is no consensus on how models and inferences are to be understood. Thus, inquiring into the nature and role of models and inferences is at the top of the philosophical agenda, and tellingly several works have been devoted to this issue in recent years (Humphreys and Imbert 2012; Meheus and Nickles 2009; Suarez 2009; Morgan and Morrison 1999).

So the attempt to understand the ways models and inferences are made basically opens two roads. The first one is to produce an analysis of the role that models and inferences play in science-how sciences use models and inferences to inquire into the world. The second one is to produce an analysis of the way models and inferences are constructed - how to model the way that scientific knowledge is pursued, especially in the light of what science tells us about our cognitive abilities.

This volume goes both ways. In the exploration of the nature and role of models and inferences, the contributed papers focus on different aspects of both the way in which models and inferences are relevant to science and the way in which science is relevant to rethinking what models and inferences are, and how models and inferences are made. In fact, the collected papers deal with issues such as: the role of the models in scientific practice; how science shapes our conceptions of models; how to model the pursuit of scientific knowledge; the relation between our conception of models and our conception of science; models and scientific explanation; models in the semantic view of theories; the applicability of mathematical models to the world; the relation between models and inferences; models as a means for acquiring new knowledge.

In dealing with those issues, the collected papers clearly underline that in order to better understand what models are it is crucial to investigate how our accounts of models and inferences are related to the way in which we analyse human knowledge, and specifically scientific knowledge.

Knowledge is indeed a crucial issue when dealing with models and inferences. To see this point let us consider some well-known and debated issues in philosophy of science.

The discussion over the nature of abduction, and the related 'Inference to the Best Explanation', that has taken place in recent years (Magnani 2009; Aliseda 2006; Lipton 2004) can be seen as an example of the relevance of the way in which inferences are analysed for the way in which science is characterized, and the reciprocal relevance of the view about science that we adopt for the definition of our ideas with regard to the nature of inferences. Whether abduction has to be considered an ampliative inference, and whether abductive reasoning has to be considered an acceptable form of scientific reasoning, are questions deeply related to the dispute over scientific realism, i.e., the way in which scientific knowledge has to be understood. 


\section{PENULTIMATE DRAFT - PLEASE CITE THE PUBLISHED VERSION}

To appear in: Models and Inferences in Science, Ippoliti, E., Sterpetti, F. and Nickles, T. (eds.), Springer.

Different ways of conceiving the same inference are due to the different conception of knowledge that one can deploy. And the concept of knowledge that one can accept is at its turn related to the way in which one conceives of the nature and the role of certain inferences.

Another example of the connection between the way in which inferences are characterized and the way in which science is analysed is the issue of the ampliativity of deduction (Prawitz 2014). To take a stance on that issue clearly makes a great difference for the way in which one conceives the scientific method. In fact, if deduction may give us new knowledge, i.e. something more than what is already contained in the premises, then the method of science may be more easily conceived of in axiomatic-deductivist terms. If, on the contrary, deduction is considered not to be ampliative, then an axiomatic-deductivist view cannot account for the process of knowledge ampliation. And subscribing to a specific view on how the scientific method has to be characterized has a great relevance for our considering deduction as ampliative or not, and thus has a great relevance on the way in which knowledge is intended (Cellucci 2013).

But even science, i.e., our recent scientific acquisitions, is relevant to logic and the way in which we conceive of the nature of inferences. For example, naturalism seems to be a mainstream tendency in contemporary philosophy, but the impact that a naturalistic stance on logic, inspired by recent work on human cognitive structures and evolution, could have on the way in which logic is conceived of is not yet clear (Schechter 2013; Dutilh Novaes 2012; Pelletier, Elio and Hanson 2008).

There is a similar relation between the way in which we conceive of mathematics and science. For example, as we have already seen above, despite the wide acceptance of the semantic view of theories, which, roughly speaking, says that a theory is the class of its models, the difficulties of making such a definition compatible with the conception of model usually accepted in model theory have not been overcome (Halvorson 2012). Moreover, models are normally understood by the semanticists as mathematical models. Thus, the problem of the relation between a theory and the world is connected to the issue of the relation between mathematics and the world. This means that the question about the role of models in science is ultimately related to the question of the nature of the relation between mathematics and the world, and thus to the question about the nature of mathematics (Cellucci 2013).

This suggests that, as in the case of logic, science not only uses mathematics, but even puts pressure on philosophers to rethink what mathematics is, so to make our conception of what mathematics is more compatible with what science tells us about the way the world is. And doing so, in turn, can even lead us to rethink what science is. Thus, not only our models and inferences, but also our way of modelling our models and inferences are worth being continuously investigated.

The papers collected in this volume are devoted precisely to the task of rethinking and better understanding what models and inferences are. It will be useful to describe their content in some detail.

Sorin Bangu's paper, On 'The Unreasonable Effectiveness of Mathematics in the Natural Sciences', deals with Eugene Wigner's famous claim that the appropriateness of the language of mathematics for the formulation of the laws of physics is a miracle 


\section{PENULTIMATE DRAFT - PLEASE CITE THE PUBLISHED VERSION}

To appear in: Models and Inferences in Science, Ippoliti, E., Sterpetti, F. and Nickles, T. (eds.), Springer.

(Wigner 1960). Bangu reconstructs Wigner's argument for the unreasonable effectiveness of mathematics and takes into account six objections to its soundness. After having shown that those six objections are weaker than it is usually thought, he raises a new objection to Wigner.

Thomas Nickles, in his Fast and Frugal Heuristics at Research Frontiers, investigates how we should model scientific decision-making at the frontiers of research. Nickles explores the applicability of Gigerenzer's 'fast and frugal' heuristics to the context of discovery. Such heuristics require only one or a very few steps to a decision and only a little information. While Gigerenzer's approach seems promising in accounting for the context of discovery, given the limited resources available in frontier contexts, it nevertheless raises challenging questions, since it seems that, according to this view of frontier epistemology, we find ourselves in the quite paradoxical situation in which the way forward may be to make sparse information even sparser.

Fabio Sterpetti's Scientific Realism, the Semantic View and Evolutionary Biology deals with the difficulties which arise when we try to apply structural realism and the semantic view of theories to some philosophical issues peculiarly related to biology. Given the central role that models have in the semantic view, and the relevance that mathematics has in the definition of the concept of model, Sterpetti focuses on population genetics, which is one of the most mathematized areas in biology, to assess French's proposal (French 2014) of adopting structural realism in dealing with biology.

Emily Grosholz's Models of the Skies examines the development of models of astronomical systems, beginning with the early 17 th century models of the solar system, and ending with late 20th century models of galaxies. More precisely, models by Kepler, Newton, Laplace, Clausius, Herschel, Rosse, Hubble, Zwicky, and Rubin are taken into account. In each case she emphasizes the distinction and the interaction between the aims of reference and analysis, and the ways in which disparate modes of representation combine to enlarge scientific knowledge.

Carlo Cellucci, in his Models of Science and Models in Science, deals with the issue of how it is possible to model science. Indeed, with regard to science, one may speak of models in two different senses, i.e. 'models of science' and 'models in science'. A model of science is a representation of how scientists build their theories, a model in science is a representation of empirical objects, phenomena, or processes. Cellucci considers five models of science: the analytic-synthetic model, the deductive model, the abstract deductive model, the semantic model, and the analytic model. After presenting them, he assesses to what extent each of them is capable of accounting for models in science.

Raffaella Campaner's Mechanistic Models and Modeling Disorders deals with the debate on how disorders should be modeled, and focuses on some issues arising from modeling neuropsychiatric disorders. More precisely, she discusses some models of attention deficit hyperactivity disorder (ADHD). The main aspects of such models are analyzed in the light of the philosophical debate about mechanistic models. The paper highlights how the neo-mechanist accounts of models can only partly capture the many aspects entering the dynamics of modeling disorders in an actual medical scenario. 


\section{PENULTIMATE DRAFT - PLEASE CITE THE PUBLISHED VERSION}

To appear in: Models and Inferences in Science, Ippoliti, E., Sterpetti, F. and Nickles, T. (eds.), Springer.

Sergio Caprara's and Angelo Vulpiani's paper, About the Ontic/Epistemic Character of Chaos and the Content of Stochastic Models in Physics, deals with the issue of clarifying the distinction between determinism and predictability. In order to show that the two concepts are completely unrelated, Caprara and Vulpiani analyse the Lyapunov exponents and the Kolmogorov-Sinai entropy and show how deterministic chaos, although it possesses an epistemic character, is not subjective at all. They also show how this is useful to shed light on the role of stochastic models in the description of the physical world.

Emiliano Ippoliti's paper, Ways of Advancing Knowledge. A Lesson from Knot Theory and Topology, investigates the ways of advancing knowledge focusing on the construction of several approaches put forward to solve problems in topology and knot theory. More precisely, Ippoliti considers two problems: the classification of knots and the classification of 3-manifolds. Examining the attempts made to solve those problems, Ippoliti is able to specify some key features of the ampliation of knowledge, such as the role of representation, theorem-proving and analogy, and to derive some considerations on the very nature of mathematical objects.

Juha Saatsi's paper, Models, Idealisations, and Realism, deals with the difficulties that, for the scientific realist, derive from the role that idealizations and abstractions play in models. Indeed, realists maintain that predictively successful models tell us the truth about the unobservable world. But how should the realist construe the way in which models latch onto unobservable reality? This is a problem, since models essentially incorporate various kinds of idealisations and approximations that cannot be interpreted realistically and that are indispensable to both their predictive and their explanatory use. Saatsi tries to face such a challenge by arguing that it is the modal character of idealisations that accounts for their utility from a realist perspective.

In Modelling Non-Empirical Confirmation Richard Dawid argues that nonempirical theory confirmation plays an important role in the scientific process and that it should be considered an extension of empirical confirmation. Since confirmation is mostly understood in Bayesian terms, Dawid proposes a formalization of nonempirical confirmation within a Bayesian framework that demonstrates that nonempirical confirmation does have the same structural characteristics of empirical theory confirmation. The No Alternative Argument (Dawid, Hartmann and Sprenger 2015 ) is then illustrated and debated.

Reuben Hersh's paper deals with the issue of modeling mathematics. Indeed, philosophy of mathematics deals with models of mathematics, which is in large part already a model, because much of mathematics is a model of physical action. Arithmetic, for instance, models the human action of counting. Hersh's suggestion is that in order to facilitate the creation of a unified field of inquiry on mathematics, philosophers should start thinking of their work as model-building instead of arguing for their chosen position against opposing positions.

Lorenzo Magnani's paper, Scientific Models Are Distributed and Never Abstract: A Naturalistic Perspective, analyses several definitions of models: from the classical ones, which see models as abstract entities and idealizations, to the more recent, which see models as fictions, surrogates, credible worlds, missing systems, makebelieve, parables, epistemic actions. Magnani reveals some of their epistemological 


\section{PENULTIMATE DRAFT - PLEASE CITE THE PUBLISHED VERSION}

To appear in: Models and Inferences in Science, Ippoliti, E., Sterpetti, F. and Nickles, T. (eds.), Springer.

inadequacies, sometimes by appealing to recent results in cognitive science. Magnani specifically addresses epistemological relying on recent results on the role of distributed and abductive cognition.

Kahindo Kamau's and Emily Grosholz's paper The Use of Models in Petroleum and Natural Gas Engineering inquires how adequate are some of the fundamental models in the science of petroleum and natural gas engineering. The authors try to unveil what assumptions were made as the models were created. They claim that a good account of the adequacy of models must be strongly pragmatist, for the questions related to their adequacy cannot be answered properly without paying attention to human purposes. They also claim that many of the distortions and oversimplifications in these models are in fact intentional and useful, when we examine the models in the light of their pragmatic aims.

\section{References}

Aliseda, A. (2006): Abductive Reasoning. Dordrecht: Springer.

Bailer-Jones, D.M. (2009): Scientific Models in Philosophy of Science. Pittsburgh: University of Pittsburgh Press.

Cellucci, C. (2013): Rethinking Logic. Logic in Relation to Mathematics, Evolution, and Meth$o d$. Dordrecht: Springer.

da Costa, N.C.A. and French, S. (2003): Science and Partial Truth. A Unitary Approach to Models and Scientific Reasoning. Oxford: Oxford University Press.

Dawid, R., Hartmann, S. and Sprenger, J. (2015): The No Alternatives Argument. The British Journal for the Philosophy of Science 66, 213-234.

Dutilh Novaes C. (2012): Formal Languages in Logic. A Philosophical and Cognitive Analysis. Cambridge: Cambridge University Press.

French, S. (2014): The Structure of the World. Metaphysics and Representation. Oxford: Oxford University Press.

French, S. (2003): A Model-Theoretic Account of Representation (Or, I Don't Know Much about Art... but I Know It Involves Isomorphism). Philosophy of Science 70, 1472-1483.

Frigg, R. and Hartmann, S. (2012): Models in Science. In: Zalta, E.N. (ed.) The Stanford Encyclopedia of Philosophy (Fall 2012 Edition), URL = $<$ http://plato.stanford.edu/archives/fall2012/entries/models-science/>.

Halvorson, H. (2012): What Scientific Theories Could Not Be. Philosophy of Science 79, 183206.

Humphreys, P. and Imbert, C. (eds.) (2012): Models, Simulations, and Representations. New York: Routledge.

Ippoliti, E. (ed.). (2014). Heuristic Reasoning. Cham: Springer.

Krause, D. and Otávio, B. (2007): Scientific Theories, Models, and the Semantic Approach. Principia 11, 187-201.

Lipton, P. (2004): Inference to the Best Explanation. London: Routledge.

Magnani, L. (2009): Abductive Cognition. The Epistemological and Eco-Cognitive Dimensions of Hypothetical Reasoning. Berlin: Springer. 


\section{PENULTIMATE DRAFT - PLEASE CITE THE PUBLISHED VERSION}

To appear in: Models and Inferences in Science, Ippoliti, E., Sterpetti, F. and Nickles, T. (eds.), Springer.

Meheus, J. and Nickles, T. (eds.) (2009): Models of Discovery and Creativity. Dordrecht: Springer.

Morgan, M.S. and Morrison, M. (eds.) (1999): Models as Mediators. Perspectives on Natural and Social Science. Cambridge: Cambridge University Press.

Morrison, M. (2009): Fictions, Representations, and Reality. In: Suárez, M. (ed.) Fictions in Science. Philosophical Essays on Modeling and Idealization. New York: Routledge, p. 110135.

Pelletier F.J., Elio R. and Hanson P. (2008). Is Logic All in Our Heads? From Naturalism to Psychologism. Studia Logica 88, 3-66.

Prawitz, D. (2014): The Status of Mathematical Knowledge. In: Ippoliti, E. and Cozzo, C. (eds.) From a Heuristic Point of View. Essays in Honour of Carlo Cellucci. Newcastle upon Tyne: Cambridge Scholars Publishing, p. 73-90.

Schechter J. (2013). Could Evolution Explain Our Reliability about Logic? In: Gendler, T.S. and Hawthorne J. (eds.) Oxford Studies in Epistemology. Volume 4. Oxford: Oxford University Press, p. 214-239.

Suarez, M. (ed.) (2009): Fictions in Science. Philosophical Essays on Modeling and Idealization. New York: Routledge.

Suppe, F. (2000): Understanding Scientific Theories: An Assessment of Developments, 19691998. Philosophy of Science 67, S102-S115.

Suppes, P. (1961): A Comparison of the Meaning and Use of Models in Mathematics and the Empirical Sciences. In: Freudenthal, J. (ed.) The Concept and the Role of the Model in Mathematics and Natural and Social Sciences. Dordrecht: Reidel, p. 163-177.

Thomson-Jones, M. (2006): Models and the Semantic View. Philosophy of Science 73, 524 535.

Wigner, E. (1960): The Unreasonable Effectiveness of Mathematics in the Natural Sciences. Communications in Pure and Applied Mathematics 13, 1-14. 